\title{
CUT STRUCTURES IN ZERO-DIVISOR GRAPHS OF COMMUTATIVE RINGS
}

\author{
M. AXTELL, N. BAETH AND J. STICKLES
}

\begin{abstract}
Zero-divisor graphs, and more recently, compressed zero-divisor graphs are well represented in the commutative ring literature. In this work, we consider various cut structures, sets of edges or vertices whose removal disconnects the graph, in both compressed and noncompressed zero-divisor graphs. In doing so, we connect these graph-theoretic concepts with algebraic notions and provide realization theorems of zero-divisor graphs for commutative rings with identity.
\end{abstract}

1. Introduction. The concept of the graph of the zero-divisors of a commutative ring was first introduced by Beck in [11] when discussing the coloring of a commutative ring. In his work, all elements of the ring were considered vertices of the graph. Since the seminal paper by D.F. Anderson and Livingston [7], the standard is to regard only nonzero zero-divisors as vertices of the graph, and we adhere to this standard.

By a ring, we mean a commutative ring with identity, typically denoted by $R$. We use $Z(R)$ to denote the set of zero-divisors of $R$ and $Z(R)^{*}$ to denote the set of nonzero zero-divisors. For any graph $G$, we denote the set of vertices of $G$ by $V(G)$ and the set of edges, nonordered pairings $a-b$ of vertices in $V(G)$, by $E(G)$. By the zero-divisor graph of $R$, denoted $\Gamma(R)$, we mean the graph with $V(\Gamma(R))=Z(R)^{*}$ and, for distinct $r, s \in Z(R)^{*}, r-s \in E(\Gamma(R))$ if and only if $r s=0$. In this case, we say $r$ and $s$ are adjacent. Similarly, one can define the compressed zero-divisor graph $\bar{\Gamma}(R)$ of a commutative ring $R$ by defining $V(\bar{\Gamma}(R))$ to be the equivalence classes of elements in $Z(R)^{*}$ defined by $[x]=[y]$ if and only if $\operatorname{ann}(x)=\operatorname{ann}(y)$. Again, $[r]-[s] \in E(\bar{\Gamma}(R))$ if

2010 AMS Mathematics subject classification. Primary 13A99.

Keywords and phrases. Commutative ring, cut vertex, cut-set, bridge, zerodivisor graph.

Received by the editors on March 26, 2012, and in revised form on April 17, 2014. 
and only if $r s=0$. For a general survey of $\Gamma(R)$, see [5]. For a survey of $\bar{\Gamma}(R)$, see [15, Section 1]. (Note that, in [15], $\bar{\Gamma}(R)$ is denoted by $\Gamma_{E}(R)$ and is referred to as the zero-divisor graph determined by equivalence classes.)

The purpose of this work is to consider cut structures, sets of edges or vertices whose removal separates (i.e., disconnects) either $\Gamma(R)$ or $\bar{\Gamma}(R)$. In Section 2, we recall the previously studied concept of cutsets in $\Gamma(R)$ and provide some new results relating these structures to algebraic properties of the ring. We then turn our attention to the concept of a bridge, or cut edge, a concept not yet studied in the context of zero-divisor graphs. At the end of this section, we give a realization theorem for $\Gamma(R)$ related to the existence of nontrivial bridges. In Section 3 we recall the compressed zero-divisor graph and show that cut-sets in $\bar{\Gamma}(R)$ correspond naturally to cut-sets in $\Gamma(R)$. We then give a realization theorem for $\bar{\Gamma}(R)$ in the presence of a nontrivial bridge. Finally, in Section 4, we return to the study of cut sets when $R$ is Noetherian. Here, we give some existence results in both $\Gamma(R)$ and $\bar{\Gamma}(R)$ in terms of $Z(R)$ written as a finite union of associated primes of $R$. Before we proceed, we recall some relevant terms from graph theory that we will use throughout.

If $x_{1}, x_{2}, \ldots, x_{n}$ are vertices in $G$, then $x_{1}-x_{2}-\cdots-x_{n}$ denotes a walk in $G$ from vertex $x_{1}$ to vertex $x_{n}$, where $x_{i}-x_{i+1} \in E(G)$ for $1 \leq i \leq n-1$. If $x_{1}, x_{2}, \ldots, x_{n}$ are distinct vertices, then the walk is called a path. A component (subgraph) of a graph is said to be connected if there exists a path between any two distinct vertices in that component. For $x, y \in V(G)$, the distance between $x$ and $y$, denoted $d(x, y)$, is the length of the shortest path between $x$ and $y$; if no path between $x$ and $y$ exists, we define $d(x, y)=\infty$. The diameter of $G$, denoted $\operatorname{diam}(G)$, is the maximum of all distances between pairs of vertices of $G$. For zero-divisor graphs, $\Gamma(R)$ is connected for all commutative rings $R$ with identity and $\operatorname{diam}(\Gamma(R)) \leq 3$ by [7, Theorem 2.3]. An analogous result for $\bar{\Gamma}(R)$ appears as [21, Proposition 1.4].

If every pair of distinct vertices are adjacent in a graph $G$, then $G$ is said to be complete, and a complete graph on $n$ vertices is denoted by $K_{n}$. An element $x \in V(G)$ is said to be looped if there exists an edge from $x$ to itself. A graph $G$ is called complete bipartite if there exist sets $A, B \subset V(G)$ with $A \cup B=V(G)$ and $A \cap B=\emptyset$ such that: 
(1) for distinct $a_{1}, a_{2} \in A$ and distinct $b_{1}, b_{2} \in B, a_{1}-a_{2} \notin E(G)$ and $b_{1}-b_{2} \notin E(G)$; and

(2) for all $a \in A$ and $b \in B, a-b \in E(G)$.

Finite complete bipartite graphs are denoted by $K_{m, n}$, where $|A|=m$ and $|B|=n$. For a general graph theory reference, see [12].

The main objects of study in this work will be those of cut-sets and bridges.

Definition 1.1. In a connected graph $G$, a set $A \subset V(G)$ is said to be a cut-set if there exist distinct $c, d \in V(G) \backslash A$ such that every path in $G$ from $c$ to $d$ involves at least one element of $A$, and no proper subset of $A$ satisfies the same condition.

Another way to define a cut-set is as a set of vertices $A$ in a connected graph $G$, where $G$ can be expressed as a union of two subgraphs $X$ and $Y$, such that:

(1) $E(X) \neq \emptyset$ and $E(Y) \neq \emptyset$,

(2) $E(X) \cup E(Y)=E(G)$ and $V(X) \cup V(Y)=V(G)$,

(3) $V(X) \cap V(Y)=A$,

(4) $X \backslash A \neq \emptyset$ and $Y \backslash A \neq \emptyset$, and

(5) no proper subset of $A$ is a cut-set for any choice of $X$ and $Y$.

In this setting, we say $A$ separates the graph into $X$ and $Y$. We note that a cut-set consisting of only one element is called a cut vertex.

Definition 1.2. For an arbitrary graph $G$, a bridge is an edge whose removal would increase the number of connected components in $G$.

Note that, if a connected graph has a bridge, then the graph formed by removing that bridge has exactly two connected components (see [12, page 46]). Moreover, if $a$ and $b$ are vertices of a graph $G$ where $a-b$ is a bridge, then each of $a$ and $b$ is either an isolated point or a cut vertex. Indeed, suppose that $a$ is not an isolated point, and let $V_{a}$ and $V_{b}$ be connected components formed by the removal of the edge $a-b$ such that $a \in V_{a}$ and $b \in V_{b}$. Since $a$ is not an isolated point, there exists $x_{a} \in V_{a}$ such that $x_{a}-a$ is an edge in $G$. Since any path from $x_{a}$ 
to $b$ must involve the edge $a-b$, the removal of vertex $a$ (and hence the edge $a-b$ ) would result in $x_{a}$ and $b$ no longer being connected. Hence, $a$ is a cut vertex. This discussion motivates the following definition.

Definition 1.3. A bridge $a-b$ of a graph $G$ is said to be nontrivial if neither $a$ nor $b$ is an isolated point, i.e., if $a$ and $b$ are both cut vertices.

2. Cut structures. In this section, we describe various algebraic properties of a commutative ring $R$ with identity such that the zerodivisor graph $\Gamma(R)$ contains a cut-set or bridge.

2.1. Cut-sets in $\Gamma(R)$. Cut-sets in the zero-divisor graph of a commutative ring were introduced in $[\mathbf{1 4}]$ and further studied in $[\mathbf{8}, \mathbf{1 0}, \mathbf{2 0}]$. Suppose that $R$ is a commutative ring and that $A$ is a cut-set separating $\Gamma(R)$ into components $X$ and $Y$. If $a \in A$ and $a$ is not adjacent to any $x \in X$, then $A \backslash\{a\}$ separates vertex sets $X \backslash\{a\}$ and $Y \cup\{a\}$, contradicting of the minimality of $A$. Therefore, $a$ is adjacent to some vertex $x_{a} \in X$ and similarly to some vertex $y_{a} \in Y$. Conversely, by the definition of $A$ being a cut-set, if there is a path $x_{a}-a-y_{a}$ in $\Gamma(R)$ with $x_{a} \in X \backslash A$ and $y_{a} \in Y \backslash A$, then $a \in A$. We summarize this in the following proposition.

Proposition 2.1. Let $R$ be a commutative ring, let $A$ separate $\Gamma(R)$ into $X$ and $Y$, and let $a \in Z(R)^{*}$. Then $a \in A$ if and only if there exist $x_{a} \in X \backslash A$ and $y_{a} \in Y \backslash A$ such that $x_{a}-a-y_{a}$ is a path in $\Gamma(R)$.

In the next several results, we address an oversight in the proof of [14, Theorem 3.3] and an overstatement found in [14, Theorem 3.4] of the same paper. In addition, we extend some of these results from finite rings to general Artinian rings. First, we give a lemma that appears in [14, Theorem 2.8].

Lemma 2.2. [14, Theorem 2.8]. Let $R \cong R_{1} \times R_{2} \times \cdots \times R_{n}$ for $n \geq 2$ with commutative rings $R_{1}, \ldots, R_{n}$, and let $A$ be a cut-set of $\Gamma(R)$. Then there exists $i \in\{1,2, \ldots, n\}$ such that if $a=\left(a_{1}, \ldots, a_{n}\right) \in A$, then $a_{k}=0$ for all $k \neq i$. 
Using this lemma, we have the following proposition which gives the existence of isolated vertices in $\Gamma\left(R_{1} \times R_{2}\right)$ for any commutative rings $R_{1}$ and $R_{2}$.

Proposition 2.3. Let $R \cong R_{1} \times R_{2}$ be a commutative ring with identity, and let $A$ be a cut-set of $\Gamma(R)$. Then, without loss of generality, $A$ isolates a vertex of the form $(r, 1)$.

Proof. By Lemma 2.2 we can, without loss of generality, assume $A=$ $\left\{(p, 0): p \in B \subseteq R_{1}^{*}\right\}$. By way of contradiction, assume that for every vertex of the form $(r, 1) \in \Gamma(R)$, there exists a vertex $(s, 0) \in Z(R)^{*} \backslash A$ such that $(r, 1)-(s, 0) \in E(\Gamma(R))$. Note that every element of $Z(R)^{*} \backslash A$ is contained in one of the following sets: $S_{1}=\left\{(0, z): z \in R_{2}^{*}\right\}$, $S_{2}=\left\{(t, 0): t \in R_{1}^{*} \backslash B\right\}, S_{3}=\left\{(w, y): w \in R_{1}^{*}, y \in Z\left(R_{2}\right)^{*}\right\}$, and $S_{4}=\left\{(x, u): x \in Z\left(R_{1}\right)^{*}, u \in R_{2}^{*}\right\}$.

Let $(0, z) \in S_{1}$. Then $(r, 1)-(s, 0)-(0, z)$ is a path in $\Gamma(R)$. Let $(t, 0) \in S_{2}$. Then $(r, 1)-(s, 0)-(0,1)-(t, 0)$ is a path in $\Gamma(R)$. If $(w, y) \in S_{3}$, there exists $y^{\prime} \in Z\left(R_{2}\right)^{*}$ such that $y y^{\prime}=0$. Thus, $(r, 1)-(s, 0)-\left(0, y^{\prime}\right)-(w, y)$ is a path in $\Gamma(R)$. Finally, consider $(x, u) \in S_{4}$. Recall that $(x, 1)$ is not isolated by $A$ via assumption. Thus, there exists $\left(x^{\prime}, 0\right) \notin A \cup\{0\}$ such that $\left(x^{\prime}, 0\right)-(x, 1) \in E(\Gamma(R))$, and hence $(r, 1)-(s, 0)-(0,1)-\left(x^{\prime}, 0\right)-(x, u)$ is a path in $\Gamma(R)$. Therefore, $\Gamma(R) \backslash A$ is connected, contradicting the fact that $A$ is a cutset.

Theorem 2.4. Let $R \cong R_{1} \times R_{2}$ be a commutative ring where $R_{2} ¥ \mathbb{Z}_{2}$. Then $A \subseteq R_{1} \times\{0\}$ is a cut-set of $\Gamma(R)$ if and only if $A=\{(s, 0)$ : $\left.s \in \operatorname{ann}(r)^{*}\right\}$, where $\operatorname{ann}(r)$ is a minimal non-trivial annihilator ideal in $R_{1}$.

Proof. Let $r \in Z\left(R_{1}\right)$ be such that $\operatorname{ann}(r)$ is a minimal non-trivial annihilator ideal, and note that $\operatorname{ann}((r, 1))=\{(s, 0): s \in \operatorname{ann}(r)\}$. Since $(r, 1)$ is isolated and $(0,1) \in V(\Gamma(R)) \backslash A$, when $A=\{(s, 0): s \in$ $\left.\operatorname{ann}(r)^{*}\right\}$ is removed, $\Gamma(R)$ is separated into two subgraphs $X$ and $Y$. By the minimality assumption on $\operatorname{ann}(r)$ and by Proposition 2.3, no proper subset of $A$ separates $\Gamma(R)$ and $A$ is a cut-set.

Conversely, let $A \subseteq R_{1} \times\{0\}$ be a cut-set of $\Gamma(R)$ isolating the vertex $(r, 1)$, where $r \in Z\left(R_{1}\right)$. Notice that, by the proof of Proposition 2.3, 
$\left\{(s, 0): s \in \operatorname{ann}(r)^{*}\right\}$ isolates $(r, 1)$, so $\left\{(s, 0): s \in \operatorname{ann}(r)^{*}\right\} \subseteq A$. Since $A$ is a cut-set of $\Gamma(R),\left\{(s, 0): s \in \operatorname{ann}(r)^{*}\right\}=A$. Notice that $\operatorname{ann}(r)$ is a minimal annihilator ideal, else $A$ is not a minimal separating set.

In particular, if $R \cong F \times R_{1}$ is a commutative ring where $F$ is a field and $R_{1} \neq \mathbb{Z}_{2}$, then $A \subseteq F \times\{0\}$ is a cut-set of $\Gamma(R)$ if and only if $A=\left\{(f, 0): f \in F^{*}\right\}$. The case where $R \cong F \times \mathbb{Z}_{2}$ is of little interest since the only cut-set in its zero-divisor graph is the cut vertex $(0,1)$.

Theorem 2.4 gives the following generalization for when $R$ is the direct product of two or more rings.

Corollary 2.5. [14, Theorem 3.3]. Let $R \cong R_{1} \times R_{2} \times \cdots \times R_{n} \times F_{1} \times$ $F_{2} \times \cdots \times F_{m}$ be a commutative ring where each $R_{i}$ is a local ring and each $F_{j}$ is a field such that $R$ is not isomorphic to $\mathbb{Z}_{2} \times F$ where $F$ is a field. Then $A$ is a cut-set of $R$ if and only if:

(i) $A=\left\{\left(0, \ldots, 0, r_{i}, 0, \ldots, 0\right) \mid r_{i} \in \operatorname{ann}(r)^{*}\right\}$, where $\operatorname{ann}(r)$ is a minimal annihilator ideal, or

(ii) $A=\left\{\left(0, \ldots, 0, f_{j}, 0, \ldots, 0\right) \mid f_{j} \in F_{j}^{*}\right\}$.

Using Corollary 2.5, we are now able to revise and extend the statement of [14, Theorem 3.4].

Theorem 2.6. Let $R$ be a non-local commutative Artinian ring, and let $A$ be a cut-set of $\Gamma(R)$. Then $A \cup\{0\}$ is an ideal of $R$.

Corollary 2.5 demonstrates that the converse of Theorem 2.6 is false. In addition, if $R$ is local, then a cut-set of $\Gamma(R)$ need not be an ideal as the following examples demonstrates.

Example 2.7. Let $R \cong \mathbb{Z}_{8}[x] /\left(x^{2}+6 x\right)$. Observe (see Figure 1 ) that $A=\{2 x, 4 x, 6 x, 2 x+4,6 x+4\}$ is a cut-set of $\Gamma(R)$, yet $A \cup\{0\}$ is not an ideal of $R$.

Notice that in Example 2.7, the cut-set $A$ does not isolate any vertices. If $A$ isolates some vertex $x$, then since a cut-set is minimal, $a x=0$ for all $a \in A$. If $x^{2} \neq 0$, then $A \cup\{0\}=\operatorname{ann}(x)$ is an ideal. If 


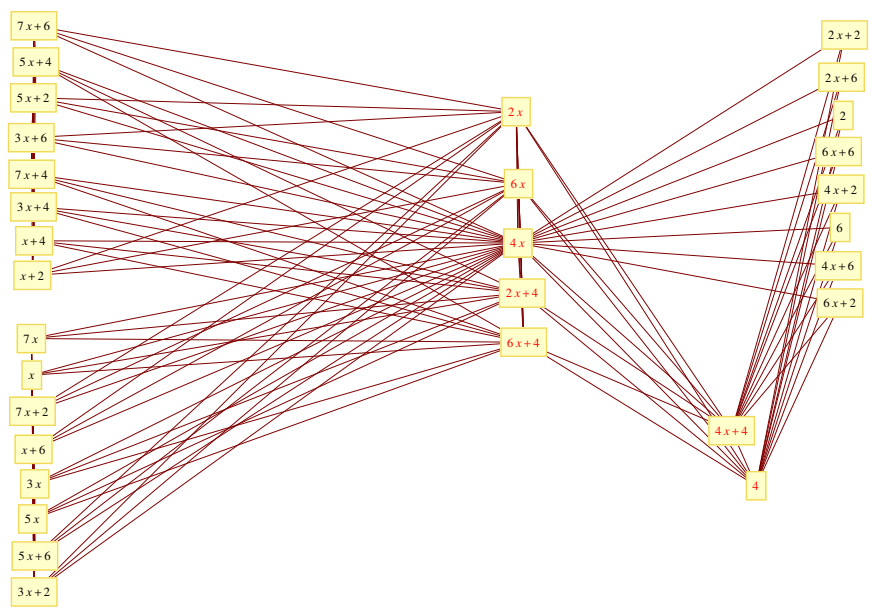

FiguRE 1. $\Gamma\left(\mathbb{Z}_{8}[x] /\left(x^{2}+6 x\right)\right)$.

$x^{2}=0$, then $A=\operatorname{ann}(x) \backslash\{x, 0\}$ and $\operatorname{ann}(x)=A \cup\{x, 0\}$. Thus, either $A \cup\{0\}$ or $A \cup\{x, 0\}$ is an annihilator ideal.

The cut-set of the zero-divisor graph of a commutative ring often demonstrates excellent algebraic structure as shown in Theorem 2.6. We now examine what algebraic structure cut-sets possess. The next proposition follows directly from Proposition 2.1.

Proposition 2.8. Let $R$ be a commutative ring with $A$ a cut-set of $\Gamma(R)$. If $a \in A$ and $\operatorname{ann}(a) \subseteq \operatorname{ann}(y)$, then $y \in A \cup\{0\}$.

The union of a cut-set $A$ with $\{0\}$ need not always be an ideal, as demonstrated in Example 2.7. However, Proposition 2.8 shows that $A \cup\{0\}$ always has the multiplicative structure of an ideal. We isolate this result in the following corollary, which also appears as $[\mathbf{1 8}$, Theorem 2.2].

Corollary 2.9. Let $R$ be a commutative ring with $A$ a cut-set of $\Gamma(R)$. For any $a \in A$ and $r \in R, r a \in A \cup\{0\}$. 
It is often the case that the vertex sets of $\Gamma(R)$ separated by a cutset also exhibit the multiplicative structure of an ideal as will be shown in Proposition 2.10. However, this is not always the case. In $\Gamma\left(\mathbb{Z}_{12}\right)$, considering the cut vertex 6 with $X=\{2,6,10\}$, we have $2 \cdot 10 \notin X$. (See Figure 2.)

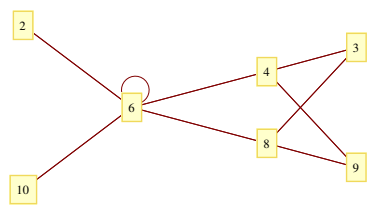

FiguRE 2. $\Gamma\left(\mathbb{Z}_{12}\right)$.

Also, in $\Gamma\left(\mathbb{Z}_{18}\right)$, considering the cut-set $A=\{6,12\}$ and $X=$ $\{3,6,12,15\}$, we have $3^{2}=15^{2}=9 \notin X$. (See Figure 3.)

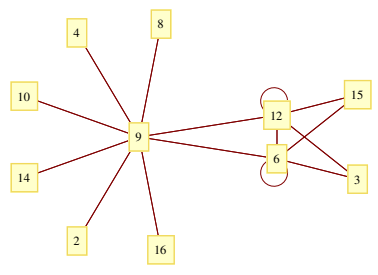

FiguRE 3. $\Gamma\left(\mathbb{Z}_{18}\right)$.

In both of these examples, the elements of $X$ which violate the multiplicative closure property are the vertices that are isolated by the cut-set. This leads to the following proposition.

Proposition 2.10. Let $R$ be a commutative ring, and let $A$ separate $\Gamma(R)$ into $X$ and $Y$. If $X$ contains no points isolated by the removal of $A$, then $X \cup\{0\}$ absorbs multiplication from $R$.

Proof. Let $c \in X \cup\{0\}$. If $c \in A \cup\{0\}$, then $c r \in A \cup\{0\} \subset X \cup\{0\}$ for all $r \in R$ by Corollary 2.9. Assume now that $c \in X \backslash A$, and let $r \in R$. If $r c=0$, we are done. If $r c \notin X \cup\{0\}$, then $r c \in Y \backslash A$. Since $c$ is not isolated by the removal of $A$, there exists $x_{c} \in X \backslash A$ such that $c x_{c}=0$. But then $x_{c} \in \operatorname{ann}(r c)$, a contradiction. 
It is then straightforward to see that, as long as non-isolated points exist for a given cut-set, we can form a subgraph $X$ such that $X \cup\{0\}$ will absorb multiplication from $R$ by placing all isolated vertices in the complementary subgraph $Y$.

Corollary 2.11. Let $R$ be a commutative ring, and let $A$ separate $\Gamma(R)$ into $X$ and $Y$. If $X$ contains no points isolated by the removal of $A$, then $X \cup\{0\}$ is multiplicatively closed.

The converse of Corollary 2.11 does not hold, as can be seen in Figure 4. Consider $\Gamma\left(\mathbb{Z}_{30}\right)$ with cut-set $A=\{6,12,18,24\}$ and $X=$ $\{6,12,18,24,5,25\}$. Clearly, $X \cup\{0\}$ is multiplicatively closed, but 5 and 25 are each isolated by the removal of $A$. Similarly, if $A=\{15\}$ and $X=\{2,4,8,14,15,16,22,26,28\}$, we see that $X \cup\{0\}$ is multiplicatively closed, but each element of $X$ (other than 15) is isolated by the removal of the cut vertex 15 .

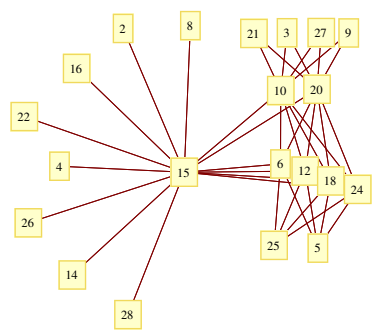

FiguRE 4. $\Gamma\left(\mathbb{Z}_{30}\right)$.

2.2. Bridges in $\Gamma(R)$. We now turn our attention to studying bridges, edges whose removal separates $\Gamma(R)$. In this section, we show that the existence of a nontrivial bridge in $\Gamma(R)$ is quite restrictive and leads to a realization theorem for $\Gamma(R)$.

In contrast to the observation made in the introduction that the vertices incident to a bridge are either cut vertices or isolated vertices, we make the following note. It is not the case that an edge connecting two cut vertices in a graph is a bridge, as can be seen in $\Gamma\left(\mathbb{Z}_{2} \times \mathbb{Z}_{2} \times \mathbb{Z}_{2}\right)$. 


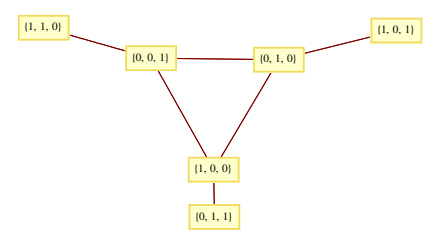

FiguRE 5. $\Gamma\left(\mathbb{Z}_{2} \times \mathbb{Z}_{2} \times \mathbb{Z}_{2}\right)$.

Recall that the diameter of the zero-divisor graph of any ring $R$ is 3 or less. The next proposition shows that diameter 0,1 or 2 cannot occur in a zero-divisor graph with a nontrivial bridge.

Proposition 2.12. Let $R$ be a commutative ring with a nontrivial bridge in $\Gamma(R)$. Then $\operatorname{diam}(\Gamma(R))=3$.

Proof. Since $\Gamma(R)$ has a nontrivial bridge $a-b, a$ is a cut vertex, and hence $2 \leq \operatorname{diam}(\Gamma(R)) \leq 3$. Assume $\operatorname{diam}(\Gamma(R))=2$, and let $\{a\}$ separate $\Gamma(R)$ into $X_{a}$ and $Y_{a}$, and $\{b\}$ separate $\Gamma(R)$ into $X_{b}$ and $Y_{b}$. Without loss of generality, $b \in X_{a}$ and $b \notin Y_{a}$. Pick $z \in X_{b}$ and $w \in Y_{b}$ such that $b \notin\{w, z\}$. Since $\operatorname{diam}(\Gamma(R))=2, z-b-w$ is a path in $X_{a}$, and, in particular, $w, z \in V\left(X_{a}\right)$. Since $Z(R)^{*}=V\left(X_{b}\right) \cup V\left(Y_{b}\right)$, we have $Z(R)^{*} \subseteq V\left(X_{a}\right)$, a contradiction. Thus, $\operatorname{diam}(\Gamma(R))=3$.

Corollary 2.13. Let $R$ be a finite commutative ring with identity such that $\Gamma(R)$ has a nontrivial bridge. Then $R$ is not local.

Proof. By [10, Theorem 2.3], if $R$ is local, then $\operatorname{diam}(\Gamma(R))=2$.

In the discussion that follows, it will be useful to consider Figure 6 , which illustrates the components of $G$ as described in the proof of Proposition 2.12.

Notation 2.14. If $\Gamma(R)$ has a nontrivial bridge $a-b$, then there are nontrivial components $X_{a}, Y_{a}, X_{b}$, and $Y_{b}$ of $\Gamma(G)$ such that $\{a\}$ separates $\Gamma(R)$ into $X_{a}$ and $Y_{a},\{b\}$ separates $\Gamma(R)$ into $X_{b}$ and $Y_{b}$, $X_{b}=X_{a} \backslash\{a\}$, and $Y_{a}=Y_{b} \backslash\{b\}$. 


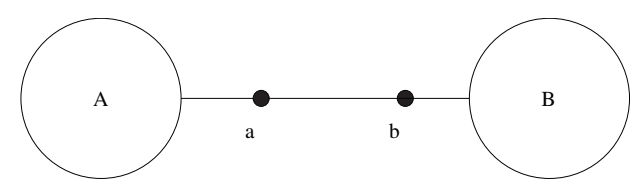

Figure 6. $A=Y_{a} \backslash\{a\}$ and $B=X_{b} \backslash\{b\}$.

Lemma 2.15. Let $R$ be a commutative ring, and let $a-b$ be a nontrivial bridge in $\Gamma(R)$. Let $X_{a}, Y_{a}, X_{b}, Y_{b}$ be as in Notation 2.14. Then:

(i) $b x=0$ for all $x \in X_{b} \backslash\{b\}$, and

(ii) $a y=0$ for all $y \in Y_{a} \backslash\{a\}$.

Proof. Let $y \in Y_{a} \backslash\{a\}$ and $x \in X_{b} \backslash\{b\}$. Since $Y_{a} \subseteq Y_{b}$, any path from $y$ to $x$ must pass through $b$. Similarly, since $X_{b} \subseteq X_{a}$, any path from $y$ to $x$ must pass through $a$. Since $\operatorname{diam}(\Gamma(R)) \leq 3$ for any ring $R$, the path must be $y-a-b-x$. Thus, $a y=0$ for all $y \in Y_{a} \backslash\{a\}$ and $b x=0$ for all $x \in X_{b} \backslash\{b\}$.

Our goal now is to prove our main result about nontrivial bridges in $\Gamma(R)$; that is, that they occur in exactly two situations.

Let $a-b$ be a nontrivial bridge in $\Gamma(R)$. Clearly $\operatorname{ann}(a) \neq \operatorname{ann}(b)$, which implies $a+b \neq 0$. If $a^{2}=0=b^{2}$, then $a-(a+b)-b$ is a path in $\Gamma(R)$, which contradicts the fact that $a-b$ is a bridge. By the paragraph preceding [8, Proposition 2.2], for every $r, s \in R \backslash Z(R)$, $r a=-s a=a$ and $r b=-s b=b$. Thus, $(r+s) a=(r+s) b=0$. Since $a-b$ is a nontrivial bridge, $r+s \in\{0, a, b\}$. By choosing $r$ and $s$ such that $r \neq-s$, we have $r+s \in\{a, b\}$. If $r+s=a$, then $a^{2}=0$. In summary, if $a-b$ a nontrivial bridge of $\Gamma(R)$ with $|R \backslash Z(R)| \geq 3$, then, without loss of generality, we have the following four properties.

(1) $a^{2}=0$ and $b^{2} \neq 0$,

(2) $r+s \in\{0, a\}$ for all $r, s \in R \backslash Z(R)$,

(3) $(r+s)^{2}=0$ for all $r, s \in R \backslash Z(R)$,

(4) $(r+s) a=(r+s) b=0$.

Suppose $r, s, t \in R \backslash Z(R)$ are distinct. Then $r+r, r+s$ and $r+t$ are three distinct elements in $\{0, a, b\}$, and (without loss of generality) 
$r+s=a$ and $r+t=b$. By property (3), $a^{2}=0$ and $b^{2}=0$, contradicting (1). Thus, we have the following proposition.

Proposition 2.16. If $R$ is a commutative ring and $\Gamma(R)$ has a nontrivial bridge, then $|R \backslash Z(R)| \leq 2$.

This rather restrictive conclusion about the number of regular elements in a ring $R$ such that $\Gamma(R)$ has a nontrivial bridge now implies that $R$ is finite.

Proposition 2.17. If $R$ is a commutative ring with identity and $\Gamma(R)$ has a nontrivial bridge, then $R \cong R_{1} \times R_{2}$, where $R_{1}$ and $R_{2}$ are finite local rings. In particular, $R$ is finite.

Proof. If $a-b$ is a nontrivial bridge in $\Gamma(R)$, then $a$ and $b$ are both cut vertices and, by Lemma $2.15, R \backslash(\operatorname{ann}(a) \cup \operatorname{ann}(b))$ is precisely the set of regular elements of $R$. By Proposition 2.16, $|R \backslash Z(R)| \leq 2$ which implies $|R|<\infty$ by [1, Theorem 2].

If $\Gamma(R)$ has a nontrivial bridge, then $R$ is not local by Corollary 2.13 . Thus, $R \cong R_{1} \times \cdots \times R_{n}$, where $n \geq 2$ and each $R_{i}$ is a finite, local ring. Assume $n \geq 3$. Since $a$ and $b$ are cut vertices, by [8, Lemma 3.1] we have, without loss of generality, that $a=(x, 0, \ldots, 0)$ and $b=(0, y, 0, \ldots, 0)$ where $x, y \neq 0$. If $c=(0,0,1,0, \ldots, 0)$, then $a-c-b$ is a path in $\Gamma(R)$, contradicting the fact that $a-b$ is a bridge. Therefore, $n=2$.

We now seek to identify the rings $R=R_{1} \times R_{2}$, with each $R_{i}$ a finite local ring, such that $\Gamma(R)$ has a nontrivial bridge. By Proposition 2.16, if $a-b$ is a nontrivial bridge in $\Gamma(R)$, then $|R \backslash Z(R)| \leq 2$. By [19, Theorem 2], if $\left(R_{i}, M_{i}\right)$ a finite local ring with identity with unique maximal ideal $M_{i}$, then $\left|R_{i}\right|=p^{n r}$ for some prime integer $p$ and positive integers $n$ and $r$ such that:

(1) $\left|Z\left(R_{i}\right)\right|=\left|M_{i}\right|=p^{(n-1) r}$, and

(2) $M_{i}^{n}=0$.

Thus, by Proposition 2.17, $\left|R_{i} \backslash Z\left(R_{i}\right)\right|=p^{n r}-p^{(n-1) r}=p^{n r-r}\left(p^{r}-\right.$ 1) $\leq 2$, which implies $p \in\{2,3\}$. By [13], the only such local rings are 
$\mathbb{Z}_{2}, \mathbb{Z}_{3}, \mathbb{Z}_{4}, \mathbb{F}_{4}$ and $\mathbb{Z}_{2}[x] /\left(X^{2}\right)$. By inspection, we obtain the following result.

Theorem 2.18. Let $R$ be a commutative ring with identity. Then $\Gamma(R)$ has a nontrivial bridge if and only if $R$ is isomorphic to $\mathbb{Z}_{2} \times \mathbb{Z}_{4}$ or $\mathbb{Z}_{2} \times \mathbb{Z}_{2}[x] /\left(x^{2}\right)$.

As a result of Theorem 2.18, we obtain the following zero-divisor graph realizability result.

Corollary 2.19. A graph $\Gamma$ with a nontrivial bridge is realizable as a zero-divisor graph of a commutative ring with identity if and only if $\Gamma \cong \Gamma\left(\mathbb{Z}_{2} \times \mathbb{Z}_{4}\right) \cong \Gamma\left(\mathbb{Z}_{2} \times \mathbb{Z}_{2}[x] /\left(x^{2}\right)\right)$.

3. Compressed zero-divisor graphs. As the reader will notice, the graph in Figure 1 is too crowded to be of much use in identifying cut-sets. Moreover, much ring-theoretic information is duplicated in the graph when vertices share the same annihilator. For this reason, the compressed zero-divisor graph of a commutative ring $R, \bar{\Gamma}(R)$, is defined. Given $a \in Z(R)^{*}$, let $[a]=\{b \in R: \operatorname{ann}(a)=\operatorname{ann}(b)\}$. Clearly, if $c, d \in[a]$, then $c$ and $d$ are adjacent to the same vertices in $\Gamma(R)$. Let $V(\bar{\Gamma}(R))=\left\{[a] \mid a \in Z(R)^{*}\right\}$, and let $[a]-[b]$ in $\bar{\Gamma}(R)$ if and only if $a-b$ in $\Gamma(R)$. Further, we place a loop on $[a]$ in $\bar{\Gamma}(R)$ if $a^{2}=0$, or equivalently, $c b=0$ for all $c, b \in[a]$. The compressed zero-divisor graph of $R$ has the benefit of simplifying $\Gamma(R)$ and making cut-sets more readily visible. See $[\mathbf{6}, \mathbf{1 5}, \mathbf{2 1}]$ for additional results on the compressed zero-divisor graph of a commutative ring.

The following useful fact appears without proof in $[\mathbf{3}, \mathbf{2 1}]$.

Lemma 3.1. For $x, y \in Z(R)^{*}$, the product $[x][y]=[x y]$ is well defined.

Proof. Note that we need only show that $\operatorname{ann}(x y)=\operatorname{ann}(a b)$ for any $a \in[x]$ and any $b \in[y]$. Suppose that $c \in \operatorname{ann}(x y)$. If $c x=0$, then $c a=0$, whence $c(a b)=0$, and finally $c \in \operatorname{ann}(a b)$. Similarly, if $c y=0$, then $c \in \operatorname{ann}(a b)$. Thus, we assume that $c x \neq 0$ and $c y \neq 0$. Since $(c x) y=0,(c x) b=0$. However, $c b \neq 0$ and $(c b) x=0$, so $(c b) a=0$. Again, $c \in \operatorname{ann}(a b)$, and we have $\operatorname{ann}(x y) \subseteq \operatorname{ann}(a b)$. A symmetric argument gives the reverse inclusion, and thus equality holds. 
There is a natural mapping from $V(\Gamma(R))$ to $V(\bar{\Gamma}(R))$ defined by $a \mapsto[a]$. It is straightforward to show that this map acts as a graph homomorphism in the sense that, if $a-b$ is $\Gamma(R)$, then either $[a]=[b]$ or $[a]-[b]$ in $V(\bar{\Gamma}(R))$.

Given a set of vertices $A$ in $\Gamma(R)$, we can define $\bar{A}=\{[a]: a \in A\}$, a set of vertices in $\bar{\Gamma}(R)$. Similarly, given a set of vertices $\bar{A}$ in $\bar{\Gamma}(R)$, we can define $A=\{b: b \in[a]$ for some $[a] \in \bar{A}\}$.

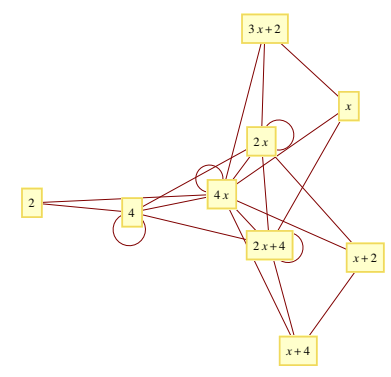

FigURE $7 . \bar{\Gamma}\left(\mathbb{Z}_{8}[x] /\left(x^{2}+6 x\right)\right)$.

3.1. Cut-sets in $\bar{\Gamma}(R)$. In this section, we study cut-sets in $\bar{\Gamma}(R)$ by way of the natural map $A \mapsto \bar{A}$ from subsets of $V(\Gamma(R))$ to subsets of $V(\bar{\Gamma}(R))$. The following result will be useful in showing that the natural projection from $V(\Gamma(R))$ to $V(\bar{\Gamma}(R))$ preserves cut-sets. Before beginning, we introduce some notation relevant for completing $r$-partite graphs. If $G$ is complete $r$-partite, then $V(G)=V_{1} \cup V_{2} \cup \cdots \cup V_{r}$, where $x-y \in E(G)$ if and only if $x \in V_{i}$ and $y \in V_{j}$ with $i \neq j$.

Theorem 3.2. Let $R \nsubseteq \mathbb{Z}_{2} \times \mathbb{Z}_{2}$ be a Noetherian commutative ring with identity, and let $r \in \mathbb{Z}^{+}$. Then:

(i) $\Gamma(R)$ is complete if and only if $\bar{\Gamma}(R) \cong K_{1}$.

(ii) For $r \geq 2, \Gamma(R)$ is complete $r$-partite if and only if $\bar{\Gamma}(R) \cong K_{2}$.

Proof.

(i) This result follows directly from [7, Theorem 2.8].

(ii) If $r=2$, consider the case when $|R|<\infty$. By [7, page 439] and [16, Theorem 1.14, Corollary 1.11], either $\Gamma(R)$ is a star graph (a graph 
isomorphic to $K_{1, m}$ where $m$ is possibly infinite) where no vertex other than the center vertex can be looped, or $\Gamma(R)$ is complete bipartite with no looped vertices. Thus $\bar{\Gamma}(R) \cong K_{2}$. Now assume $|R|=\infty$. If $\Gamma(R)$ is a star graph, then only the center vertex may be looped by [10, Theorem 4.1], and thus $\bar{\Gamma}(R) \cong K_{2}$. If $\Gamma(R)$ is not a star graph, then by [16, Theorem 1.14], $R$ is isomorphic to a subring of a direct product of two integral domains. Then no vertices in $\Gamma(R)$ are looped, and $\bar{\Gamma}(R) \cong K_{2}$.

Now assume $r \geq 3$. By [2, Theorem 3.1], if $\Gamma(R)$ is complete $r$ partite, then $\left|V_{i}\right|>1$ for at most one $i \in\{1,2, \ldots, r\}$, and if $V_{j}=\{x\}$, then $x^{2}=0$. Moreover, by [2, Theorem 3.6], $R$ is isomorphic to a subring of $F \times S$, where $F$ is a field and $S$ is a finite ring. Since $S$ is a finite commutative ring with identity, $S$ can be expressed as a product of finite fields and finite local rings. For convenience, we write $R \cong T_{1} \times T_{2}$, where $T_{1}$ is the direct product of the fields, and $T_{2}$ is the direct product of the finite local rings.

We claim that if $V=V_{i}$ for some $i$ where $|V|>1$, then $x^{2} \neq 0$ for all $x \in V$. First note that, if $x \in V_{j}$ for some $j \neq i$, then $x=\left(0, x^{\prime}\right)$ for some $x^{\prime} \in T_{2}-\{0\}$, else $x^{2}=0$. Assume that $(t, z)^{2}=0$ for some $(t, z) \in V$. Then $t=0$ and $z^{2}=0$. Since $|V|>1$, there exists $(u, w) \in V$ distinct from $(0, z)$. Since $r \geq 3$, there exists $(0, x) \notin V$ with $x^{2}=0$ and $x z=0$. Now consider the vertex $(0, x+z)$ of $\Gamma(R)$. Since $(0, z)(0, x+z)=(0,0)$ and $(0, x+z) \neq(0, x),(0, x+z) \notin V$ as distinct vertices in $V$ cannot be adjacent. Since $(u, w) \in V$ and $(0, z+x) \notin V,(0, z+x)(u, w)=(0,0)$, and hence $z w+x w=0$. Since $x w=0, z w=0$. and thus $(0, z)(u, w)=(0,0)$, contradicting the fact that both $(0, z)$ and $(u, w)$ are in $V$. Therefore, $V$ contains no element that squares to zero.

Since $x^{2} \neq 0$ for all $x \in V$ and $\Gamma(R)$ is complete $r$-partite, $\operatorname{ann}(x)=$ $\operatorname{ann}(y)$ for all $x, y \in V$. Clearly, if $v, w \in V(\Gamma(R)) \backslash V$, then $\operatorname{ann}(v)=$ $\operatorname{ann}(w)$, and thus $\bar{\Gamma}(R) \cong K_{2}$.

Conversely, suppose that $\bar{\Gamma}(R) \cong K_{2}$, and let $[x]$ and $[y]$ be the two distinct vertices in $\bar{\Gamma}(R)$. If $x^{2}=y^{2}=0$, then $\operatorname{ann}(x)=\operatorname{ann}(y)=$ $Z(R)$, and hence $[x]=[y]$, a contradiction. If $x^{2} \neq 0$ and $y^{2} \neq 0$, then $\operatorname{ann}(x)^{*}=[y]$ and $\operatorname{ann}(y)^{*}=[x]$. Then $\Gamma(R)$ is complete bipartite. If, without loss of generality, $x^{2}=0$ and $y^{2} \neq 0$, then $\operatorname{ann}(y)^{*}=[x]$ and $\operatorname{ann}(x)=Z(R)$. Then $\Gamma(R)$ is complete $r$-partite for some $r \geq 2$. 
Observe that $r>2$ if and only if $|[x]|>1$.

By [21, Proposition 1.5], if $\bar{\Gamma}(R) \cong K_{n}$, then $n \in\{1,2\}$. Thus, Theorem 3.2 classifies which $\Gamma(R)$ occur when $\bar{\Gamma}(R)$ is a complete graph.

We give two lemmas that further clarify the structure of cut-sets, both of which will be useful in proving Theorem 3.6 concerning the connection between the cut-sets in $\Gamma(R)$ and cut-sets in $\bar{\Gamma}(R)$.

Lemma 3.3. Let $R$ be a commutative ring with identity, let $A$ be a cutset of $\Gamma(R)$ and let $A^{\prime}=\{v \in V(\Gamma(R))$ : $\operatorname{ann}(v)=\operatorname{ann}(a)$ for some $a \in$ $A\}$. Then $A=A^{\prime}$.

Proof. Let $A$ separate $\Gamma(R)$ into $X$ and $Y$. Clearly, $A \subseteq A^{\prime}$, so suppose that $b \in A^{\prime}$. Then $\operatorname{ann}(b)=\operatorname{ann}(a)$ for some $a \in A$. By Proposition 2.1, there exist $x_{a} \in X$ and $y_{a} \in Y$ such that $x_{a}-a-y_{a}$. Since $\operatorname{ann}(b)=\operatorname{ann}(a)$, we have $x_{a}-b-y_{a}$. Again, by Proposition 2.1, $b \in A$.

Lemma 3.4. Let $R$ be a commutative ring with identity, and let $A$ be a cut-set of $\Gamma(R)$. If $[b]=[c]$ for all $b, c \in V(\Gamma(R)) \backslash A$, then $\bar{\Gamma}(R) \cong K_{2}$ (or equivalently, $\Gamma(R)$ is complete $r$-partite for some $r \geq 2$ ).

Proof. Clearly, if $A$ is a cut-set of $\Gamma(R)$, then $\bar{\Gamma}(R) \nRightarrow K_{1}$. Suppose $[b]=[c]$ for all $b, c \in V(\Gamma(R)) \backslash A$. Then $V(\bar{\Gamma}(R))=\bar{A} \cup\{[b]\}$. If $b \in \operatorname{ann}(b)$, then $b x=0$ for all $x \in Z(R)^{*} \backslash A$, and $A$ would not be a cut-set of $\Gamma(R)$. Therefore, it must be the case that $b \notin \operatorname{ann}(b)$. Thus, $\operatorname{ann}(b) \subset A \cup\{0\}$ and, by the minimality condition of cut-sets, $\operatorname{ann}(b)=A \cup\{0\}$. This means that $\Gamma(R)$ consists of the cut-set $A$ and a collection of vertices each isolated by the removal of $A$. By way of contradiction, we assume $\bar{\Gamma}(R) \nRightarrow K_{2}$, in which case $\bar{\Gamma}(R)$ has three or more vertices. We consider two cases.

If $\operatorname{ann}(b)$ is not a maximal annihilator ideal, then there exists $0 \neq d \in R$ such that $\operatorname{ann}(b) \subsetneq \operatorname{ann}(d)$. This implies that $Z(R)=\operatorname{ann}(d)$ since $\Gamma(R)$ consists of the cut-set $A$ and a collection of vertices each connected to every element of $A$ and to no other vertex. In particular, $Z(R)$ is an ideal. Let $[a] \neq[c]$ with $[a],[c] \in \bar{A}$. Since $Z(R)$ is an ideal, we have $0 \neq a+b \in Z(R)$. Since $b^{2} \neq 0$, we must have $a+b \notin A$. By assumption, $[a+b]=[b]$, so $c(a+b)=0$, which gives 
$c a=0$. Thus, $[c][a]=0$, implying that $\bar{\Gamma}(R)$ is complete. However, by [21, Proposition 1.5], $\bar{\Gamma}(R)$ cannot be complete if $|V(\bar{\Gamma}(R))| \geq 3$, a contradiction.

If $\operatorname{ann}(b)$ is a maximal annihilator ideal, then by [9, Corollary 3.5 , Proposition 3.6] either $Z(R)=\operatorname{ann}(b)$, or $Z(R)=\operatorname{ann}(b) \cup \operatorname{ann}(a)$, where $\operatorname{ann}(a)$ is a maximal annihilator ideal and $\operatorname{ann}(b) \cap \operatorname{ann}(a)=\{0\}$. Since $b^{2} \neq 0, Z(R) \neq \operatorname{ann}(b)$. Thus, $Z(R)=\operatorname{ann}(b) \cup \operatorname{ann}(a)$ with $a \in A$. Since $\bar{\Gamma}(R)$ is not complete, there exists $[c] \neq[a]$ in $\bar{A}$ such that $[a][c] \neq 0$. Since $b^{2} \neq 0$, we see $b \in \operatorname{ann}(c) \backslash \operatorname{ann}(b)$, and thus, $\operatorname{ann}(c) \subseteq \operatorname{ann}(a)$. Now, for each $[q] \in \bar{A}$, we must have that $[a][q] \neq 0$ because $\operatorname{ann}(b) \cap \operatorname{ann}(a)=\{0\}$. Furthermore, since $\operatorname{ann}(c) \subseteq \operatorname{ann}(a)$, it must be the case that $[c][q] \neq 0$. Therefore, no two vertices in $\bar{A}$ are adjacent, and so $\bar{\Gamma}(R)$ is a star-graph with center $[b]$. Since $\bar{\Gamma}(R)$ has at least three vertices, $[b]$ is a cut vertex of $\bar{\Gamma}(R)$ with at least two ends. By [15, Proposition 6.9], $\operatorname{ann}(b)$ is the only associated prime of $R$, contradicting the fact that there are two maximum annihilator ideals. Therefore, $\bar{\Gamma}(R) \cong K_{2}$.

Remark 3.5. In the last case of the proof of Lemma 3.4, we saw that when $Z(R)^{*}$ is the disjoint union of two annihilator ideals with one a maximal annihilator ideal, $\bar{\Gamma}(R)$ must be a star graph. We will return to this idea in Proposition 4.3 when we characterize $\Gamma(R)$ in this setting.

In Figure 3, the compressed zero-divisor graph of $\mathbb{Z}_{8}[x] /\left(x^{2}+6 x\right)$ is shown, and it is easily noted that $\{[4 x],[2 x],[2 x+4]\}$ does in fact form a cut-set. This set of compressed elements corresponds to $A=\{2 x, 4 x, 6 x, 2 x+4,6 x+4\}$, which is a cut-set of $\Gamma\left(\mathbb{Z}_{8}[x] /\left(x^{2}+6 x\right)\right)$. This is not a coincidence as Theorem 3.6 indicates. A similar result for finite rings appears in [22]. We remark that the assumption $\bar{\Gamma}(R) \neq K_{2}$ is to ensure that $\bar{\Gamma}(R)$ does indeed have a cut-set.

Theorem 3.6. Let $R$ be a Noetherian commutative ring with identity, and assume $\bar{\Gamma}(R) \neq K_{2}$. Let $A \subseteq V(\Gamma(R))$, and set $A^{\prime}=\{v \in$ $V(\Gamma(R)):[v] \in \bar{A}\}$.

(i) If $A$ is a cut-set of $\Gamma(R)$, then $\bar{A}$ is a cut-set of $\bar{\Gamma}(R)$.

(ii) If $\bar{A}$ is a cut-set in $\bar{\Gamma}(R)$, then $A^{\prime}$ is a cut-set in $\Gamma(R)$. 
Proof. We begin by proving (ii). Since $\bar{A}$ is a cut-set of $\bar{\Gamma}(R)$, there exist distinct, non-adjacent $[x],[y] \in V(\bar{\Gamma}(R)) \backslash \bar{A}$ such that, for any path $[x]-\left[z_{1}\right]-\cdots-\left[z_{n}\right]-[y]$ in $\bar{\Gamma}(R),\left[z_{i}\right] \in \bar{A}$ for some $i \in\{1, \ldots, n\}$. Observe that $x y \neq 0$ in $R$ since $[x]$ is not adjacent to $[y]$ in $\bar{\Gamma}(R)$. If $A^{\prime}$ does not separate $x$ and $y$ in $\Gamma(R)$, there exists a path $x-b_{1}-\cdots-b_{n}-y$ in $\Gamma(R)$ such that $b_{i} \notin A^{\prime}$ for all $i \in\{1, \ldots, n\}$. Then $[x]-\left[b_{1}\right]-\cdots-\left[b_{n}\right]-[y]$ is a walk in $\bar{\Gamma}(R) \backslash \bar{A}$, contradicting the fact that $\bar{A}$ separates $[x]$ and $[y]$ in $\bar{\Gamma}(R)$. Thus, $A^{\prime}$ separates $x$ and $y$ in $\Gamma(R)$.

Now assume that $A^{\prime}$ is not a minimal separating set in $\Gamma(R)$, that is, there exists a cut-set $B \subsetneq A^{\prime}$ of $\Gamma(R)$ separating two distinct vertices $w$ and $v$. In other words, every path from $w$ to $v$ in $\Gamma(R)$ passes through a vertex of $B$. By definition, $w, v \notin B$.

Assume $[w]=[v]$. Then $\operatorname{ann}(w)=\operatorname{ann}(v)$. Since $B$ separates $w$ and $v$ and since $\operatorname{ann}(w)=\operatorname{ann}(v)$, we see that $\operatorname{ann}(w)^{*} \subseteq B$. Since $B$ is a cut-set, $\operatorname{ann}(w)^{*}=B$ by minimality. If $[w] \in \bar{B}$, then $\operatorname{ann}(w)=\operatorname{ann}(b)$ for some $b \in B$, and hence $w \in B$ by Proposition 2.8, a contradiction. We may thus assume that $[w] \notin \bar{B}$. Since $\operatorname{ann}(w)^{*}=B, \bar{B}$ isolates $[w]$. Since $\bar{B} \cup\{[w]\} \neq V(\bar{\Gamma}(R)), \bar{B}$ separates $\bar{\Gamma}(R)$. But $\bar{B} \subseteq \bar{A}$ and $\bar{A}$ is a cut-set. By minimality, $\bar{B}=\bar{A}$. Let $a \in A^{\prime} \backslash B$, and consider $[a] \in \bar{A}$. By construction, $[w]-[a]$ is an edge in $\bar{\Gamma}(R)$ and $[w] \neq[a]$ (since $[a] \in \bar{A}$ and $[w] \notin \bar{A})$. Then $w-a \in E(\Gamma(R))$, and because $\operatorname{ann}(w)=\operatorname{ann}(v)$, $w-a-v$ is a path in $\Gamma(R)$. This contradicts the fact that $B$ separates $w$ and $v$.

Thus, we may assume that $[w] \neq[v]$. If $[w] \in \bar{B}$, then $\operatorname{ann}(w)=$ $\operatorname{ann}(b)$ for some $b \in B$. However, $B$ is a cut-set, and by Proposition 2.8, $w \in B \cup\{0\}$, a contradiction. A similar argument applies to $[v]$, and thus $[w],[v] \notin \bar{B}$. If $\bar{B} \subsetneq \bar{A}$, then $\bar{B}$ is not a cut-set, so there exists a path $[w]-\left[x_{1}\right]-\cdots-\left[x_{n}\right]-[v]$ in $\bar{\Gamma}(R)$ with $\left[x_{i}\right] \notin \bar{B}$ for all $i \in\{1, \ldots, n\}$. Then, in $\Gamma(R), w-x_{1}-\cdots-x_{n}-v$ is a path with $x_{i} \notin B$, for all $i \in\{1, \ldots, n\}$, a contradiction to the fact that $B$ is a cut-set. Therefore, $\bar{B}=\bar{A}$.

We claim now that $B=A^{\prime}$. If not, let $a \in A^{\prime} \backslash B$. Since $\bar{B}=\bar{A}$, $[a] \in \bar{B}$. Then $\bar{B} \backslash\{[a]\}$ is not a cut-set and does not separate $\bar{\Gamma}(R)$.

We will show that $B$ does not separate $\Gamma(R)$. Pick distinct $c, d \in$ $V(\Gamma(R)) \backslash B$, and assume $c d \neq 0$. If $[c]=[d]$, choose $[l] \notin \bar{B} \backslash\{[a]\}$ such that $[c]-[l]$ is an edge in $\bar{\Gamma}(R)$ (else $\bar{B} \backslash\{[a]\}$ isolates $[c]$ ). Then $c-l-d$ 
is a path in $\Gamma(R)$. If $[l]=[a]$, then $c-a-d$ is a path in $\Gamma(R)$ avoiding $B$. If $[l] \neq[a]$, then $c-l-d$ is a path in $\Gamma(R)$ avoiding $B$. If $[c] \neq[d]$, then there exists a path $[c]-\left[x_{1}\right]-\cdots-\left[x_{n}\right]-[d]$ with no $\left[x_{i}\right]$ in $\bar{B} \backslash\{[a]\}$. Thus, $c-x_{1}-\cdots-x_{n}-d$ is a path in $\Gamma(R)$ with $x_{i}=a$ whenever $\left[x_{i}\right]=[a]$. By construction, $x_{i} \notin B$ for each $i \in\{1, \ldots, n\}$. Then, $B$ does not separate $\Gamma(R)$, a contradiction. Thus, $B=A^{\prime}$ and $A^{\prime}$ is a cut-set.

We now prove (i). Let $A$ be a cut-set of $\Gamma(R)$. Thus, by Lemma 3.3, $A=A^{\prime}$. Assume $\bar{A}$ in $V(\bar{\Gamma}(R))$ is not a cut-set. Then either $\bar{A}$ is not a minimal separating set of $\bar{\Gamma}(R)$ or $\bar{A}$ does not properly contain a cut-set and is not a cut-set itself.

First assume that $\bar{A}$ separates $\bar{\Gamma}(R)$ but is not a minimal separating set of $\bar{\Gamma}(R)$, and let $\bar{B} \subsetneq \bar{A}$ be a cut-set of $\bar{\Gamma}(R)$. By (ii), $B=\{v \in$ $V(\Gamma(R)):[v] \in \bar{B}\}$ is a cut-set of $\Gamma(R)$ and $B \subsetneq A^{\prime}$. Thus $A=A^{\prime}$ is not a cut-set of $\Gamma(R)$, a contradiction.

Now assume $\bar{A}$ is not a cut-set of $\bar{\Gamma}(R)$ and does not properly contain one. Since $A=A^{\prime}$ is a cut-set of $\Gamma(R)$, by Lemma 3.4, we can choose $b, c \in V(\Gamma(R)) \backslash A^{\prime}$ with distinct $[b],[c] \notin \bar{A}$. Then, since $\bar{A}$ is not a cut-set, either $[b]-[c]$ is an edge in $\bar{\Gamma}(R)$ or $[b]-\left[x_{1}\right]-\cdots-\left[x_{n}\right]-[c]$ is a path in $\bar{\Gamma}(R)$ with no $\left[x_{i}\right] \in \bar{A}$. Then, either $b-c$ is an edge in $\Gamma(R)$ or $b-x_{1}-\cdots-x_{n}-c$ is a path in $\Gamma(R)$ with no $x_{i} \in A^{\prime}$, whence $A^{\prime}$ does not separate $\Gamma(R)$. Since $A=A^{\prime}, A$ is not a cut-set of $\Gamma(R)$, a contradiction.

Remark 3.7. Note that Theorem 3.6 does not guarantee that, for a given set $A$ in $V(\Gamma(R))$, if $\bar{A}$ is a cut-set of $\bar{\Gamma}(R)$, then $A$ is a cut-set of $\Gamma(R)$. In $\Gamma\left(\mathbb{Z}_{12}\right)$, we see $A=\{4\}$ is not a cut-set. However, $\bar{A}=\{[4]\}$ is a cut-set in $\bar{\Gamma}\left(\mathbb{Z}_{12}\right)$. (See Figures 8 and 9.)

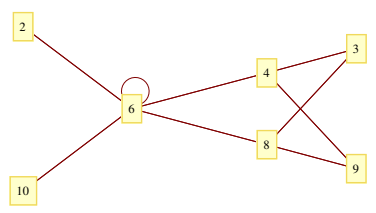

Figure 8. $\Gamma\left(\mathbb{Z}_{12}\right)$. 


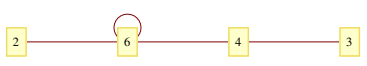

Figure 9. $\bar{\Gamma}\left(\mathbb{Z}_{12}\right)$.

3.2. Bridges in $\bar{\Gamma}(R)$. We now return to the study of bridges. The following two results give a statement analogous to Theorem 2.18 for compressed zero-divisor graphs containing nontrivial bridges.

Theorem 3.8. Let $R$ be a commutative ring with identity such that $\bar{\Gamma}(R)$ has a nontrivial bridge. Then $\bar{\Gamma}(R)$ is isomorphic to the line graph $[c]-[a]-[b]-[d]$ in which only the vertex $[a]$ is looped.

Proof. Let $[a]-[b]$ be the nontrivial bridge in $\bar{\Gamma}(R)$. Then both vertices $[a]$ and $[b]$ are cut vertices in $\bar{\Gamma}(R)$. By [3, Corollary 4.3], ann $(a)$ and $\operatorname{ann}(b)$ are associated primes of $R$. By [15, Proposition $5.9]$, there is exactly one vertex $[c]$ (necessarily of degree one) adjacent to $[a]$ and exactly one vertex $[d]$ of degree one adjacent to $[b]$. Again, by [15, Proposition 5.9], $\bar{\Gamma}(R)$ has exactly four vertices, whence $\bar{\Gamma}(R)$ is $[c]-[a]-[b]-[d]$.

Suppose that neither $[a]$ nor $[b]$ is looped, and consider the nonzero zero-divisor $c d$. Since $a(c d)=0$ and $b(c d)=0,[c d] \notin\{[a],[b],[c],[d]\}$, an impossibility. Thus, at least one of $[a]$ or $[b]$ is looped. However, if $[a]$ and $[b]$ are both looped, then $a^{2}=b^{2}=0$. Since $[a] \neq[b], a+b$ is a nonzero zero-divisor not in $[a] \cup[b]$, lest $c(a+b)=0$ or $d(a+b)=0$. But then $[a]-[a+b]-[b]$ is a path in $\bar{\Gamma}(R)$, contradicting the fact that $[a]-[b]$ is a bridge. Without loss of generality, we assume that $[a]$ is looped and $[b]$ is not looped.

If we assume that $[d]$ is looped, then $d^{2}=0$ and $b+d$ is a nonzero zero-divisor. Since $a d \neq 0,[b+d] \notin\{[a],[b],[c]\}$. Since $b^{2} \neq 0$, $[b+d] \neq[d]$. Therefore, $[d]$ is not looped. We can also see that $[c]$ is not looped by showing that, if $c^{2}=0$, then $[a+b] \notin\{[a],[b],[c],[d]\}$. Therefore, $\bar{\Gamma}(R)$ is the graph $[c]-[a]-[b]-[d]$ in which only vertex $[a]$ is looped.

Using the structure of $\bar{\Gamma}(R)$ given in Theorem 3.8, we can now determine when $\bar{\Gamma}(R)$ has a nontrivial bridge if $R$ is Artinian. 
Theorem 3.9. Let $R$ be an Artinian ring. Then $\bar{\Gamma}(R)$ has a nontrivial bridge if and only if $R$ is isomorphic to $F \times S$, where $F$ is a field and $S$ is a local ring (not a field) with maximal ideal $M$ such that $M^{2}=0$.

Proof. If $R \cong F \times S$, where $F$ is a field and $S$ is a local ring with maximal ideal $M$ such that $M^{2}=0$, then each zero-divisor of $R$ resides in exactly one of the following equivalence classes: $[(1, z)]$, $[(0, z)],[(1,0)],[(0,1)]$, where $z$ denotes a fixed, nonzero zero-divisor of $S$. Note also that $\bar{\Gamma}(R)$ is precisely $[(1, z)]-[(0, z)]-[(1,0)]-[(0,1)]$ where only the vertex $[(0, z)]$ is looped.

Conversely, assume that $\bar{\Gamma}(R)$ has a nontrivial bridge. By Theorem 3.8, $\bar{\Gamma}(R)$ is isomorphic to a line graph with four vertices. Since $R$ is Artinian, $R$ can be written as $R \cong R_{1} \times R_{2} \times \cdots \times R_{n}$, where each $R_{i}$ is a local ring. If $n=1$ and $M$ is the maximal ideal of $R_{1}$, then $M^{m}=0$ for some $m \geq 1$ since $R_{1}$ is Artinian. If $m=1$, the $R_{1}$ is a field, and $V(\Gamma(R))=\emptyset$. If $m \geq 2$, then $\operatorname{diam}(\Gamma(R))<3$, which gives that $\Gamma(R)$ does not have a nontrivial bridge. If $n \geq 3$, then by $[8$, Lemma 2.1], $\Gamma(R)$ has at least three cut-sets. By Corollary 2.5, every cut-set of $\Gamma(R)$ has the form $A=0 \times 0 \cdots \times 0 \times \operatorname{ann}\left(a_{i}\right)^{*} \times 0 \cdots \times 0$ for some nonzero $a_{i} \in R_{i}$. Then, if $A \neq A^{\prime}$ for two cut-sets $A$ and $A^{\prime}$ of $\Gamma(R)$, then $\bar{A} \neq \overline{A^{\prime}}$ are distinct cut-sets of $\bar{\Gamma}(R)$ by Theorem 3.6. That is, $\bar{\Gamma}(R)$ has at least three cut-sets. Both of these conclusions contradict the fact that $\bar{\Gamma}(R)$ is a line graph with four vertices.

If $R_{1}$ and $R_{2}$ are both fields, then $\Gamma(R)$ is complete bipartite, and hence, $\bar{\Gamma}(R)$ has only two vertices. If neither $R_{1}$ nor $R_{2}$ is a field, then there exist at least five vertices $[(1,0)],[(0,1)],[(0, x)],[(y, 0)],[(w, z)]$ in $\bar{\Gamma}(R)$ where $w, x \in Z\left(R_{1}\right)^{*}$ and $y, z \in Z\left(R_{2}\right)^{*}$. Therefore, it must be the case that, without loss of generality, $R_{1}$ is a field and $R_{2}$ is a local Artinian ring that is not a field. Thus, $M^{m}=0$ for some integer $m \geq 2$. If $M^{2} \neq 0$ and $M^{m}=0$ for some $m>2$, then $\bar{\Gamma}(R)$ has at least six vertices $[(1,0)],[(0,1)],[(0, z)],\left[\left(0, z^{m-1}\right)\right],[(1, z)],\left[\left(1, z^{m-1}\right)\right]$ for some $z \in Z\left(R_{2}\right)^{*}$. Therefore, $R$ is isomorphic to $F \times S$, where $F$ is a field and $S$ is a local ring with maximal ideal $M$ such that $M^{2}=0$.

We note that $R$ need not be Artinian for $\bar{\Gamma}(R)$ to have a nontrivial bridge. Indeed, if $F$ and $K$ are fields, then let $R=F \times$ $K\left[X_{1}, X_{2}, X_{3}, \ldots\right] / M^{2}$ where $M=\left(X_{1}, X_{2}, X_{3}, \ldots\right)$. Then $\bar{\Gamma}(R)$ is the line graph $\left[\left(1, x_{1}\right)\right]-\left[\left(0, x_{1}\right)\right]-[(1,0)]-[(0,1)]$ in which only the 
vertex $\left[\left(0, x_{1}\right)\right]$ is looped. However, we do not know of an example of a non-Artinian ring $S$ not isomorphic to the direct product of a field and a local ring $(S, M)$ with $M^{2}=0$ such that $\bar{\Gamma}(R)$ has a nontrivial bridge.

It is clear that, if $\Gamma(R)$ has a nontrivial bridge, then $\bar{\Gamma}(R)$ also has a nontrivial bridge. However, if $\bar{\Gamma}(R)$ has a nontrivial bridge $[a]-[b]$, then the corresponding edge set in $\Gamma(R)$ need not be a nontrivial bridge in $\Gamma(R)$ though it is a minimal set of edges whose removal separates $\Gamma(R)$ into two distinct connected components. Clearly, the image of such a minimal separating set of edges in $\Gamma(R)$ need not even be well-defined, let alone separate $\bar{\Gamma}(R)$. Conversely, the pre-image of a minimal separating set of edges in $\bar{\Gamma}(R)$ need not be a minimal separating set of edges in $\Gamma(R)$. The ring $\mathbb{Z}_{12}$ can be used to illustrate both of these observations. In summary, locating a bridge in a zerodivisor graph gives the existence of cut vertices in that same graph, but there is no hope in even partially reversing this process.

Figures 8 and 9 also show that though the endpoints of a nontrivial bridge are cut vertices, the incident endpoints of a minimal set of edges whose removal separates $\Gamma(R)$ need not form a cut-set of $\Gamma(R)$. These incident endpoints can fail to separate $\Gamma(R)$, or may separate but fail to be a minimal such set of vertices. In summary, there is a nice correspondence between cut-sets in $\Gamma(R)$ and cut-sets in $\bar{\Gamma}(R)$, but no such result can be formulated for sets of cut-edges.

4. Noetherian rings. Recall that in a Noetherian ring $R, Z(R)=$ $\bigcup_{i=1}^{n} \operatorname{ann}\left(x_{i}\right)$, where the $\operatorname{ann}\left(x_{i}\right)$ are precisely the associated prime ideals (see [17, Theorem 80]). In this section, we investigate how cut-sets and $\operatorname{Ass}(R)$ are related. We assume $R$ is Noetherian, and to simplify the statements of some results, we set the following notation for the sequel.

Notation 4.1. Let $R$ be a Noetherian ring and write

$$
Z(R)=\bigcup_{i=1}^{n} \operatorname{ann}\left(x_{i}\right) \quad \text { with } \quad \operatorname{Ass}(R)=\left\{\operatorname{ann}\left(x_{i}\right)\right\}
$$


Set

$$
A=\bigcup_{i=1}^{n} A_{i}
$$

where, for each $i \in\{1, \ldots, n\}$,

$$
A_{i}=\left\{y_{i, \lambda}: \operatorname{ann}\left(y_{i, \lambda}\right)=\operatorname{ann}\left(x_{i}\right)\right\}_{\lambda \in \Lambda_{i}}
$$

is indexed by some (possibly infinite) set $\Lambda_{i}$.

The following proposition is easily seen to be equivalent to $[\mathbf{9}$, Proposition 3.6].

Proposition 4.2. Let $Z(R)=P_{1} \cup P_{2}$ where $P_{1}$ and $P_{2}$ are incomparable prime ideals (maximal in $Z(R)$ ). Then:

(i) $\operatorname{diam}(\Gamma(R))=2$ if and only if $P_{1} \cap P_{2}=\{0\}$.

(ii) $\operatorname{diam}(\Gamma(R))=3$ if and only if $P_{1} \cap P_{2} \neq\{0\}$.

We now give several equivalent conditions equivalent to that given in Proposition 4.2 (i) in the case when $P_{1}$ and $P_{2}$ are both annihilator ideals.

Proposition 4.3. Let $Z(R)=\operatorname{ann}\left(x_{1}\right) \cup \operatorname{ann}\left(x_{2}\right)$ where both are prime (annihilator ideals) and incomparable. The following are equivalent:

(i) $\operatorname{ann}\left(x_{1}\right) \cap \operatorname{ann}\left(x_{2}\right)=\{0\}$,

(ii) $\operatorname{diam}(\Gamma(R))=2$,

(iii) $A=Z(R)^{*}$,

(iv) $\Gamma(R)$ is complete bipartite with disjoint vertex sets $\operatorname{ann}\left(x_{1}\right)^{*}$ and $\operatorname{ann}\left(x_{2}\right)^{*}$.

Proof. The equivalence of (1) and (2) follows from Proposition 4.2.

We now show that (1) implies both (3) and (4). With $i \in\{1,2\}$, if $x_{i} \in \operatorname{ann}\left(x_{i}\right)^{*}$, then $x_{i} \notin \operatorname{ann}\left(x_{j}\right)$ for $j \in\{1,2\} \backslash\{i\}$. Then $d(x, y)=2$. Also, there is some $z \in Z(R)^{*}$ such that $x_{1}-z-x_{2}$ a path in $\Gamma(R)$. This implies $z \in \operatorname{ann}\left(x_{1}\right) \cap \operatorname{ann}\left(x_{2}\right)=\{0\}$, a contradiction. Thus, $x_{1} \in \operatorname{ann}\left(x_{2}\right)$ and $x_{2} \in \operatorname{ann}\left(x_{1}\right)$.

Let $y \in \operatorname{ann}\left(x_{1}\right)^{*}$ and $z \in \operatorname{ann}\left(x_{2}\right)^{*}$. Since $d(y, z) \leq 2$, either $y z=0$, or there exists $w \in Z(R)^{*}$ with $y-w-z$ a path in $\Gamma(R)$. If $y z \neq 0$, 
then $y w \in \operatorname{ann}\left(x_{2}\right)$ and $w z \in \operatorname{ann}\left(x_{1}\right)$. Since $\operatorname{ann}\left(x_{i}\right)$ is prime for each $i \in\{1,2\}, w \in \operatorname{ann}\left(x_{1}\right) \cap \operatorname{ann}\left(x_{2}\right)$, a contradiction. Thus, $y z=0$ for all $y \in \operatorname{ann}\left(x_{1}\right)$ and $z \in \operatorname{ann}\left(x_{2}\right)$.

Finally, without loss of generality, let $y, z \in \operatorname{ann}\left(x_{1}\right)$. If $y z=0$, then since each $\operatorname{ann}\left(x_{i}\right)$ is prime, either $y \in \operatorname{ann}\left(x_{2}\right)$, or $z \in \operatorname{ann}\left(x_{2}\right)$, a contradiction. Thus, $\Gamma(R)$ is complete bipartite with disjoint vertex sets $\operatorname{ann}\left(x_{1}\right)^{*}$ and $\operatorname{ann}\left(x_{2}\right)^{*}$ and $A=Z(R)^{*}$.

We now show that (3) implies (2). Clearly, $\operatorname{diam}(\Gamma(R))>1$, and we can choose distinct $y, z \in Z(R)^{*}$. If, without loss of generality, $\operatorname{ann}(y)=\operatorname{ann}(z)=\operatorname{ann}\left(x_{1}\right)$, then either there is $c \in \operatorname{ann}\left(x_{1}\right)^{*} \backslash\{y, z\}$, which gives a path $y-c-z$ in $\Gamma(R)$, or $\operatorname{ann}\left(x_{1}\right) \subseteq\{0, y, z\}$, in which case $y-z \in E(\Gamma(R))$. Either way, $d(y, z) \leq 2$.

Now, suppose $\operatorname{ann}(y)=\operatorname{ann}\left(x_{1}\right)$ and $\operatorname{ann}(z)=\operatorname{ann}\left(x_{2}\right)$. If $y z \neq 0$, $y \in \operatorname{ann}\left(x_{1}\right)=\operatorname{ann}(y)$ and $z \in \operatorname{ann}\left(x_{2}\right)=\operatorname{ann}(z)$. Then $y(y z)=0=$ $(y z) z$ and $d(y, z) \leq 2$.

That (4) implies (2) is clear.

Corollary 4.4. Let $Z(R)=\operatorname{ann}\left(x_{1}\right) \cup \operatorname{ann}\left(x_{2}\right)$ where $\operatorname{ann}\left(x_{1}\right) \cap$ $\operatorname{ann}\left(x_{2}\right)=\{0\}$. Then there exist exactly two cut-sets in $\Gamma(R)$, namely, $\operatorname{ann}\left(x_{1}\right)^{*}$ and $\operatorname{ann}\left(x_{2}\right)^{*}$.

In the finite case, we have the following.

Corollary 4.5. Let $R$ be a finite ring. If $Z(R)=\operatorname{ann}\left(x_{1}\right) \cup \operatorname{ann}\left(x_{2}\right)$, where $\operatorname{ann}\left(x_{1}\right) \cap \operatorname{ann}\left(x_{2}\right)=\{0\}$, then $R \cong F_{1} \times F_{2}$, where $F_{1}$ and $F_{2}$ are finite fields.

Proof. Let $x \in Z(R)^{*}$. If $x^{2}=0$, then $x \in \operatorname{ann}\left(x_{1}\right) \cap \operatorname{ann}\left(x_{2}\right)$, a contradiction. Thus, $\Gamma(R)$ has no looped vertices. By [10, Corollary 4.6], $R \cong F_{1} \times F_{2}$, where $F_{1}$ and $F_{2}$ are finite fields.

The next result provides some insight into the structure of $\Gamma(R)$.

Proposition 4.6. Let

$$
Z(R)=\bigcup_{i=1}^{n} \operatorname{ann}\left(x_{i}\right),
$$


where the $\operatorname{ann}\left(x_{i}\right)$ are non-comparable prime ideals with $\left|A_{i}\right|=\left|\Lambda_{i}\right|$, where each $\Lambda_{i}$ is a (possibly infinite) index set for $A_{i}$. Then $\Gamma(R)$ contains the graph $K_{\left|\Lambda_{1}\right|, \ldots,\left|\Lambda_{n}\right|}$ as a subgraph. In particular, $y_{i, \alpha}$ is adjacent to $y_{i^{\prime}, \beta}$ for each pair of distinct $i$ and $i^{\prime}$ and for all $\alpha$ and all $\beta$. Moreover, $y_{i, \alpha}$ is adjacent to $y_{i, \beta}$ for all $\alpha$ and $\beta$ if and only if $x_{i}^{2}=0$.

Proof. Fix $l, m \in\{1, \ldots, n\}$, and let $z \in \operatorname{ann}\left(x_{l}\right) \backslash \operatorname{ann}\left(x_{m}\right)$. Then $x_{l} z=0 \in \operatorname{ann}\left(x_{m}\right)$. Since $\operatorname{ann}\left(x_{m}\right)$ is prime, $x_{l} \in \operatorname{ann}\left(x_{m}\right)$. Since this holds for all $l \in\{1, \ldots, n\} \backslash\{m\}$, we see that $y_{i, \alpha}$ is adjacent to $y_{i^{\prime}, \beta}$ for any $i, i^{\prime}, \alpha, \beta$ with $i \neq i^{\prime}$. If $y_{i, \alpha}$ is adjacent to $y_{i, \beta}$ for some $\alpha$ and $\beta$, then $y_{i, \alpha} \in \operatorname{ann}\left(x_{i}\right)$. Since $\operatorname{ann}\left(y_{i, \alpha}\right)=\operatorname{ann}\left(x_{i}\right), x_{i} \in \operatorname{ann}\left(x_{i}\right)$, so $x_{i}^{2}=0$. If $x_{i}^{2}=0$, then $x_{i} \in \operatorname{ann}\left(x_{i}\right)=\operatorname{ann}\left(y_{i, \alpha}\right)$ for each $\alpha$, and thus $y_{i, \alpha}$ is adjacent to $y_{i, \beta}$ for any $\alpha, \beta$.

The next result generalizes Corollary 4.4 in the case where $|\operatorname{Ass}(R)|$ $>2$.

Proposition 4.7. Let $R$ be a Noetherian ring with

$$
Z(R)=\bigcup_{i=1}^{n} \operatorname{ann}\left(x_{i}\right),
$$

where the $\operatorname{ann}\left(x_{i}\right)$ are non-comparable prime ideals. Let $X$ be a cut-set of $\Gamma(R)$ separating the graph into $W$ and $Z$.

(i) For each $i$, either $A_{i} \subseteq X$ or $A_{i} \cap X=\emptyset$.

(ii) $A_{i} \subseteq X$ for some $i$.

(iii) If there exist distinct $A_{i}$ and $A_{j}$ not contained in $X$, then, without loss of generality, $A_{k} \subseteq W$ for all $A_{k} \varsubsetneqq X$.

(iv) If there exists a unique $A_{i}$ such that $A_{i}$ is not in $X$, then, without loss of generality, either $A_{i} \subseteq W$, or each element of $A_{i}$ is isolated by $X$.

\section{Proof.}

(i) Let $a, b \in A_{i}$ be such that $a \in X$ and $b \notin X$. Then any path through $a$ can be redirected to a path through $b$ since $\operatorname{ann}(a)=\operatorname{ann}(b)=\operatorname{ann}\left(x_{i}\right)$. Thus, $X \backslash\{a\}$ contains a cut-set, contradicting the minimality of $X$. 
(ii) Suppose $X$ contains no $A_{i}$. Then by (i), $X \cap A_{i}=\emptyset$ for all $i$. Let $w \in W$ and $z \in Z$. All paths from $w$ to $z$ involve elements of $X$, and, without loss of generality, we can choose $w \in \operatorname{ann}\left(x_{1}\right)$ and $z \in \operatorname{ann}\left(x_{i}\right)$. By Proposition 4.6, either $w-x_{1}-x_{i}-z$, or $w-x_{1}-z$ is a path in $\Gamma(R)$, a contradiction.

(iii) Suppose that $a, b \in A_{i}$ with $a \in W$ and $b \in Z$. Pick $c \in A_{j}$. If $c \in W$, then $c-b \in E(\Gamma(R))$ by Proposition 4.6, a contradiction since $X$ is a cut-set. A similar argument shows $c \in Z$. Thus, $A_{i}$ (similarly $A_{j}$ ) is contained in either $W$ or $Z$. Assume $A_{i} \subseteq W$ and $A_{j} \subseteq Z$. Then $x_{1}-x_{2} \in E(\Gamma(R)$, which contradicting that $X$ is a cut-set.

(iv) Assume that $A_{i} \not \subset X$ for some $i \in\{1, \ldots, n\}$ and that $A_{j} \subseteq X$ for all $j \in\{1, \ldots, n\} \backslash\{i\}$. If $A_{i}^{2}=\{0\}$, then, without loss of generality, $A_{i} \subseteq W$ since $a-b \in E(\Gamma(R))$ for all $a, b \in A_{i}$. Thus, we may assume that $A_{i}^{2} \neq\{0\}$. Clearly, $\operatorname{ann}\left(x_{i}\right)^{*}$ is a set which isolates every vertex of $A_{i}$. If $\operatorname{ann}\left(x_{i}\right) \subseteq X$, then, by the minimality of $X$, we have that $\operatorname{ann}\left(x_{i}\right)=X$, and the result holds. If $\operatorname{ann}\left(x_{i}\right) \nsubseteq X$, then there exists $y \in \operatorname{ann}\left(x_{i}\right) \backslash X$. If there exists $a, b \in A_{i}$ with $a \in W$ and $b \in Z$, then $a-y-b$ is a path from $W$ to $Z$ not through $X$, a contradiction. Thus, $A_{i}$ is contained in either $W$ or $Z$.

Note that proper containment in Proposition 4.7 (ii) is possible. Consider $R=\mathbb{Z}_{8}[x] /\left(x^{2}\right)$. Then $\operatorname{ann}(4 x)$ is a maximal annihilator ideal (in fact, $Z(R)^{*}=\operatorname{ann}(4 x)$ ), but $\{4 x\}$ is not a cut-set. Also, even though $\{4 x, 2 x, 6 x, 4,2 x+4,4 x+4,6 x+4\}$ is a cut-set, it does not correspond to any annihilator ideal (maximal or otherwise).

We conclude by stating two corollaries achieved by examining the situations in Propositions 4.6 and 4.7 when situated within the compressed zero-divisor graph. In Corollary 4.9, we omit statement (i) since it gives no interesting information.

Corollary 4.8. Let $Z(R)=\bigcup_{i=1}^{n} \operatorname{ann}\left(x_{i}\right)$, where the $\operatorname{ann}\left(x_{i}\right)$ are noncomparable prime ideals with $\left|A_{i}\right|=\left|\Lambda_{i}\right|$, where each $\Lambda_{i}$ is a (possibly infinite) index set for $A_{i}$. Then $\bar{\Gamma}(R)$ contains the graph $K_{n}$. Moreover, the vertex $\left[x_{i}\right]$ looped if and only if $x_{i}^{2}=0$. 


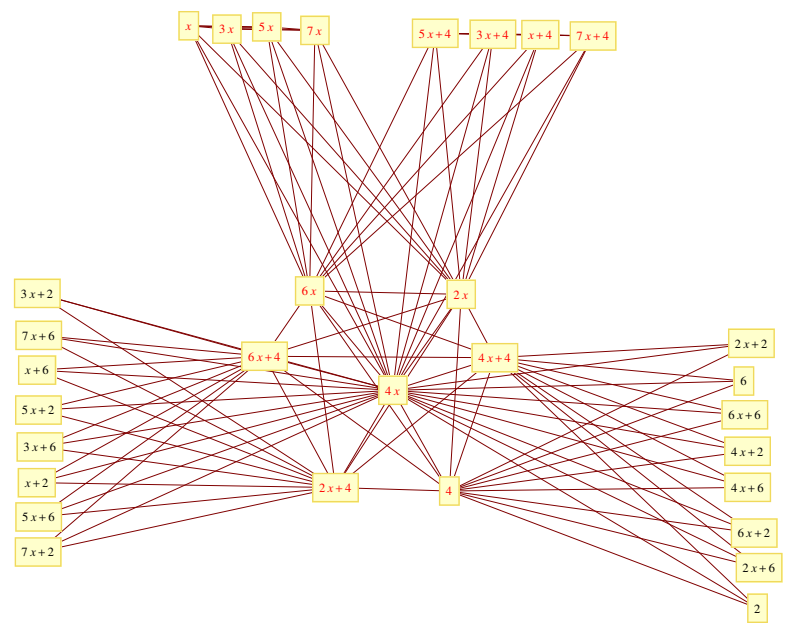

Figure 10. $\Gamma\left(\mathbb{Z}_{8}[x] /\left(x^{2}\right)\right)$.

Corollary 4.9. Let $R$ be a Noetherian ring with

$$
Z(R)=\bigcup_{i=1}^{n} \operatorname{ann}\left(x_{i}\right),
$$

where the $\operatorname{ann}\left(x_{i}\right)$ are non-comparable prime ideals. Let $\bar{X}$ be a cut-set of $\bar{\Gamma}(R)$ separating the graph into $\bar{W}$ and $\bar{Z}$.

(ii) $\left[x_{i}\right] \in \bar{X}$ for some $i$,

(iii) if there exist distinct $\left[x_{i}\right],\left[x_{j}\right] \notin \bar{X}$, then, without loss of generality, $\left[x_{k}\right] \subseteq \bar{W}$ for all $\left[x_{k}\right] \varsubsetneqq \bar{X}$, and

(iv) if there exists a unique $\left[x_{i}\right]$ such that $\left[x_{i}\right] \notin \bar{X}$, then, without loss of generality, either $\left[x_{i}\right] \subseteq \bar{W}$, or $\left[x_{i}\right]$ is isolated by $\bar{X}$.

\section{REFERENCES}

1. S. Akbari, D. Kiani, F. Mohammadi and S. Moradi, The total graph and regular graph of a commutative ring, J. Pure Appl. Alg. 213 (2009), 2224-2228.

2. S. Akbari, H.R. Maimani and S. Yassemi, When a zero-divisor graph is planar or a complete r-partite graph, J. Alg. 270 (2003), 169-180. 
3. B. Allen, E. Martin, E. New and D. Skabelund, Diameter, girth and cut vertices of the graph of equivalence classes of zero-divisors, Involve 5 (2012), 5160.

4. D.D. Anderson and M. Naseer, Beck's coloring of a commutative ring, J. Alg. 159 (1993), 500-514.

5. D.F. Anderson, Michael Axtell and J. Stickles, Zero-divisor graphs in commutative rings, in Commutative algebra, Noetherian and non-Noetherian perspectives, Springer-Verlag, New York, 2011.

6. D.F. Anderson and J. LaGrange, Commutative Boolean monoids, reduced rings, and the compressed zero-divisor graph, J. Pure Appl. Alg. 216 (2012), 16261636.

7. D.F. Anderson and P. Livingston, The zero-divisor graph of a commutative ring, J. Alg. 217 (1999), 434-447.

8. M. Axtell, N. Baeth and J. Stickles, Cut vertices in zero-divisor graphs of finite commutative rings, Comm. Alg. 39 (2011), 2179-2188.

9. M. Axtell, J. Coykendall and J. Stickles, Zero-divisor graphs of polynomials and power series over commutative rings, Comm. Alg. 33 (2005), 2043-2050.

10. M. Axtell, J. Stickles and W. Trampbachls, Zero-divisor ideals and realizable zero-divisor graphs, Involve 2 (2009), 17-27.

11. I. Beck, Coloring of commutative rings, J. Alg. 116 (1988), 208-226.

12. G. Chartland, Introductory graph theory, Dover Publications, Inc., New York, 1977.

13. B. Corbas, Rings with few zero-divisors, Math. Ann. 157 (1964), 215-218.

14. B. Cote, C. Ewing, M. Huhn, C.M. Plaut and D. Weber, Cut-sets in zerodivisor graphs of finite commutative rings, Comm. Alg. 39 (2011), 2849-2861.

15. J. Coykendall, S. Sather-Wagstaff, L. Sheppardson and S. Spiroff, On zerodivisor graphs, Progr. Comm. Alg 2, Walter de Gruyter, Berlin, 2012.

16. F. DeMeyer and K. Schneider, Automorphisms and zero-divisor graphs of commutative rings, Int. J. Comm. Rings 1 (2002), 93-106.

17. I. Kaplansky, Commutative rings, Polygonal Publishing House, Washington, 1974 .

18. H.R. Maimani, M. Mogharrab and S. Yassemi, Complete r-partite zerodivisor graphs and coloring of commutative semigroups, arXiv:math/0701627.

19. R. Raghavendran, Finite associative rings, Comp. Math. 21 (1969), 195229 .

20. S. Redmond, Cut vertices and degree one vertices of zero-divisor graphs, Comm. Alg. 40 (2012), 2749-2756.

21. S. Spiroff and C. Wickham, A zero divisor graph determined by equivalence classes of zero divisors, Comm. Alg. 39 (2011), 2338-2348.

22. D. Weber, Zero-divisor graphs and lattices of finite commutative rings, RoseHulman Undergrad. Math. J. 12 (2011), 58-70. 
Department of Mathematics, University of St. Thomas, St. Paul, MN 55105 Email address: axte2004@stthomas.edu

Department of Mathematics and Computer Science, University of Central Missouri, WARrensburg, MO 64093

Email address: baeth@ucmo.edu

Department of Mathematics, Millikin University, Decatur, IL 62522

Email address: jstickles@millikin.edu 